\title{
Quantum dot-based immunofluorescent imaging of Ki67 and identification of prognostic value in HER2-positive (non-luminal) breast cancer
}

This article was published in the following Dove Press journal:

International Journal of Nanomedicine

II March 2014

Number of times this article has been viewed

\author{
Jin-Zhong Sun ${ }^{1, *}$ \\ Chuang Chen ${ }^{1, *}$ \\ Guan Jiang² \\ Wei-Qun Tian ${ }^{3}$ \\ Yan $\mathrm{Li}^{4}$ \\ Sheng-Rong Sun' \\ 'Department of Breast and Thyroid \\ Surgery, Renmin Hospital of Wuhan \\ University, Wuhan, Hubei, People's \\ Republic of China; ${ }^{2}$ Department \\ of Dermatology, Renmin Hospital \\ of Wuhan University, Wuhan, \\ Hubei, People's Republic of China; \\ ${ }^{3}$ Department of Biomedical \\ Engineering, Wuhan University \\ School of Basic Medical Sciences, \\ Wuhan, Hubei, People's Republic of \\ China; ${ }^{4}$ Department of Oncology, \\ Zhongnan Hospital of Wuhan \\ University and Hubei Key Laboratory \\ of Tumor Biological Behaviors and \\ Hubei Cancer Clinical Study Center, \\ Wuchang District, Wuhan, Hubei, \\ People's Republic of China
}

*These authors contributed equally to this work

Correspondence: Sheng-Rong Sun Department of Breast and Thyroid Surgery, Renmin Hospital of Wuhan University, Tower 238 Jiefang Road, Wuhan, Hubei Province, 430060,

People's Republic of China

Tel +86278804 I9II extn 82012

Emailsun137@sina.com
Background: The immunohistochemical assessment of Ki67 antigen (Ki67) is the most widely practiced measurement of breast cancer cell proliferation; however, it has some disadvantages and thus the prognostic value of Ki67 in breast cancer remains controversial. Our previous studies confirmed the advantages of quantum dots-based nanotechnology for quantitative analysis of biomarkers compared with conventional immunohistochemistry (IHC). This study was designed to assess Ki67 by quantum dot-immunohistochemistry (QD-IHC) and investigate the prognostic value of the Ki67 score in human epidermal growth factor receptor 2 (HER2)positive (non-luminal) breast cancer.

Methods: Ki67 expression in 108 HER2-positive (non-luminal) breast cancer specimens was detected by IHC and QD-IHC. Two observers assessed the Ki67 score independently and comparisons between the two methods were made. The prognostic value of the Ki67 score for five-year disease-free survival was estimated.

Results: The same antigen localization, high correlation of staining rates $(r=0.993)$, and high agreement of measurements ( $\kappa=0.874$ ) of Ki67 expression (cutoff: $30 \%$ ) in breast cancer were found by QD-IHC and conventional IHC. The QD-IHC had a better interobserver agreement for the Ki67 score than conventional IHC $(t=-7.280, P<0.01)$. High Ki67 expression (cutoff: $30 \%$ ) was associated with shorter disease-free survival (log-rank test; IHC, $P=0.026$; QD-IHC, $P=0.001$ ), especially in the lymph node-negative subgroups (log-rank test; IHC, $P=0.017$; QD-IHC, $P=0.002$ ).

Conclusion: QD-IHC imaging of Ki67 was an easier and more accurate method for detecting and assessing Ki67. The Ki67 score was an independent prognosticator in the HER2-positive (non-luminal) breast cancer patients.

Keywords: quantum dots, breast cancer, Ki67, disease-free survival, prognosis

\section{Introduction}

Breast cancer is the most common cancer and the leading cause of cancer death in females worldwide. ${ }^{1}$ Understanding its biological behavior and identifying objective prognosticators are very important for personalized treatment. Ki67 was identified by Gerdes et al in several studies as a nuclear nonhistone protein which expressed in all phases of the cell cycle except the G0 phase. ${ }^{2-4}$ Compared with other biomarkers, Ki67 is an ideal proliferation marker in breast cancer. Many studies have demonstrated the primary role of proliferation signatures in breast cancer and most of them support the prognostic value of Ki67. .6 For example, a gene expression-based intrinsic subtype classification of breast cancers with pathological features found that a Ki67 score $\geq 14 \%$ distinguished luminal B from luminal A. ${ }^{7}$ This was subsequently supported by the 
St Gallen 2011 Expert Panel as an alternative to molecular subtyping. ${ }^{8}$ Similarly, an algorithm based on semiquantitative scores for estrogen receptor (ER) and progesterone receptor (PR) expression, human epidermal growth factor receptor 2 (HER2) status, and the Ki67 score derived from immunohistochemistry (IHC) staining (IHC-4) showed similar prognostic performance in the 21-gene recurrence score for ER-positive breast cancer. ${ }^{9}$ However, the clinical prognostic value of the Ki67 score in HER2-positive (nonluminal; defined as HER2 overexpressed or amplified, ER and PR absent $)^{8}$ breast cancer remains unknown.

The immunohistochemical assessment of the proportion of cells staining for the nuclear antigen Ki67 is the most widely used method in clinical samples. ${ }^{10}$ However, this method has some disadvantages, such as susceptibility to interfering factors, unstable sensitivity, high discrepancy among laboratories, subjective interpretation, and no unified judgment, thereby severely limiting its clinical utility. ${ }^{11}$ Therefore, a sensitive, accurate, economical, and convenient method for Ki67 detection is urgently required.

Quantum dots (QDs), a new semiconductor nanocrystal, have unique photophysical properties, such as size-tunable symmetric emission bands, superior light absorbance, high fluorescent intensity, and strong photostability. ${ }^{12}$ Their unique optical properties have led to QD-based nanotechnology being expanded into a wide variety of biomedical applications, such as cancer diagnosis, monitoring, pathogenesis, treatment, molecular pathology, and heterogeneity in combination with cancer biomarkers; QD-based nanotechnology has the potential for wide application, especially in the field of in vitro cancer molecular pathology. ${ }^{13}$ Our previous study of molecular targeted imaging of cancer cells and molecules demonstrated the advantages of QD-based molecular pathology, ${ }^{14-18}$ such as superior fluorescent efficiency over organic fluorescent dyes, better signal clarity, and higher sensitivity, and accuracy compared with conventional IHC techniques.

This study was designed to assess Ki67 using QDimmunohistochemistry (QD-IHC) and investigate the prognostic value of the Ki67 score in HER2-positive (nonluminal) breast cancer.

\section{Materials and methods}

\section{Patients and specimens}

Formalin-fixed, paraffin-embedded specimens of 108 HER2positive (non-luminal) (HER2 IHC 3+ or fluorescence in situ hybridization [FISH] amplification, ER and PR IHCnegative) invasive breast cancer patients from January 2006 to
September 2008, aged from 32 to 80 (median 46) years, were collected from the Renmin Hospital of Wuhan University, People's Republic of China. All patients with stage I to stage III breast cancer who had undergone six to eight cycles of doxorubicin-based chemotherapy after breast surgery were eligible; none of them had received molecular targeted therapy. Of note, in the People's Republic of China, the vast majority of the patients do not receive targeted therapy, for economic reasons. The patients with stage II to stage III breast cancer received radiotherapy after chemotherapy. Major pathological parameters were available, including tumor size, location, and number, lymph node status, histological grade, and ER, PR, and HER2 status, as determined by conventional IHC. Major treatment information, including types of surgery and adjuvant treatments (chemotherapy and radiotherapy), was obtained from the medical records of each patient. All patients were on a regular follow-up schedule. Written informed consent was obtained from the patients and the Ethics Committee of Renmin Hospital of Wuhan University approved the study protocol. Patient characteristics are summarized in Table 1. The whole study process is outlined in Figure 1.

The primary endpoint was disease-free survival (DFS), defined as the time interval from breast cancer surgery to the first evidence of recurrence (local, regional, or distant). If there was no recurrence, patients were censored on the last follow-up. In this study, we only selected the 5-year data for analysis.

\section{Ki67 testing and assessment}

The primary antibody used was a mouse anti-human monoclonal antibody against Ki67 (Clone: MIB1, 1:100 dilution; Dako Denmark A/S, Glostrup, Denmark); biotinylated horse anti-mouse immunoglobulin $\mathrm{G}$ ( $\operatorname{IgG}$ ) (1:400 dilution) was

Table I Characteristics of the patients

\begin{tabular}{ll}
\hline Items & Number (\%) \\
\hline Age (years) & \\
$\leq 50$ & $66(6 I . I I)$ \\
$>50$ & $42(38.89)$ \\
Pathological tumor size (cm) & \\
PTI $(T \leq 2)$ & $52(48.15)$ \\
PT2 $(2<T \leq 5)$ & $46(42.59)$ \\
PT3 $(T>5)$ & $10(9.26)$ \\
Pathological node status & \\
Negative & $53(49.07)$ \\
Positive & $55(50.93)$ \\
Tumor grade & \\
Grade I & $15(13.89)$ \\
Grade 2 & $5 \mathrm{I}(47.22)$ \\
Grade 3 & $42(38.89)$ \\
\hline
\end{tabular}




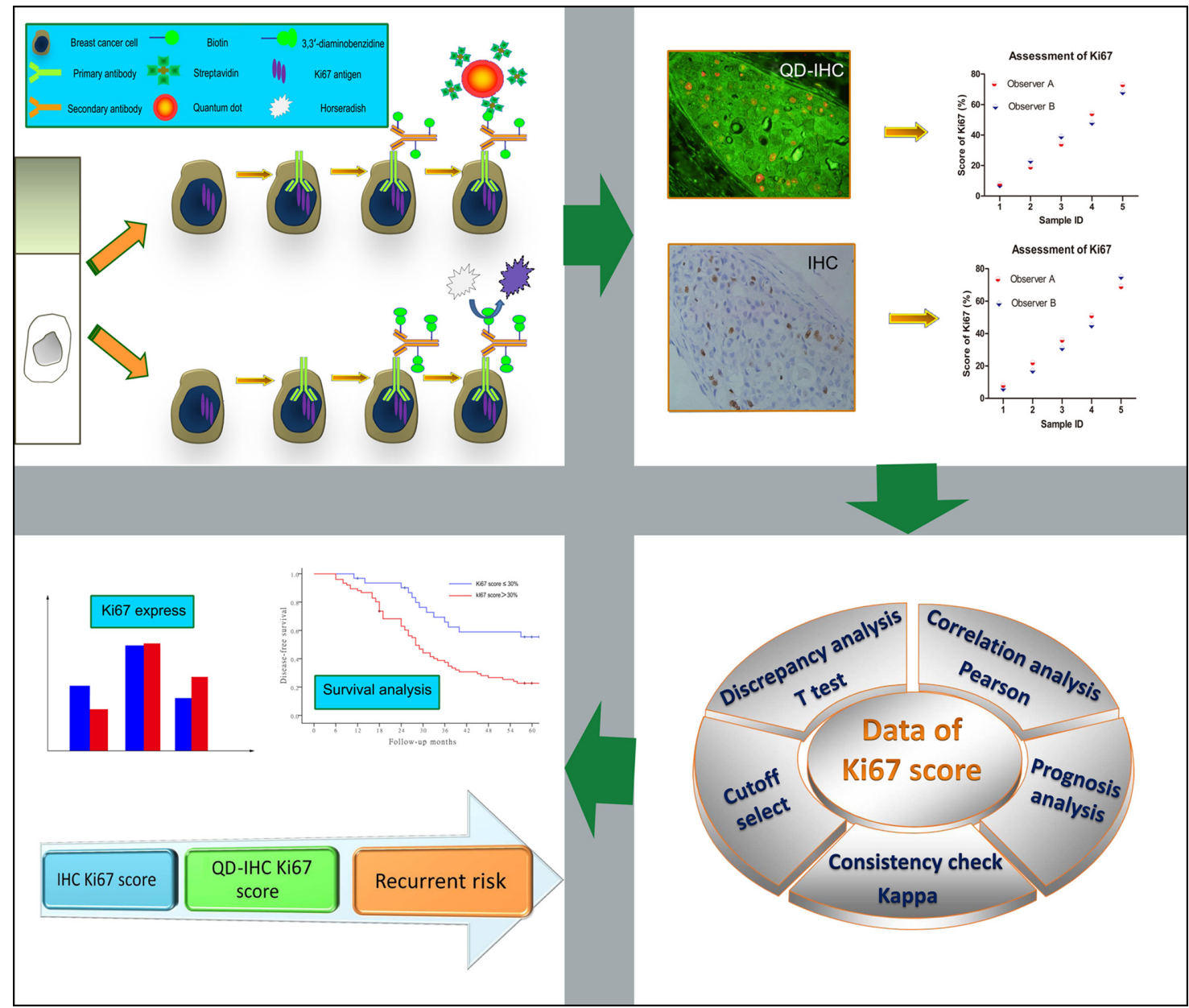

Figure I Flow chart of the study design.

Notes: Ki67 expression in the breast cancer specimens was detected by QD-IHC and IHC. Two observers assessed the Ki67 score independently. The two methods were analyzed and compared. Finally, Ki67 expression and 5-year disease free survival were analyzed.

Abbreviations: IHC, immunohistochemistry; QD-IHC, quantum dot-immunohistochemistry.

used as the secondary antibody. QD-conjugated streptavidin (QD-SA) probes, with an emission wavelength of 605 $\mathrm{nm}$ were kindly provided by Wuhan Jiayuan Quantum Dot Technological Development Co, Ltd. (Wuhan, People's Republic of China). Three-to-seven serial sections (4 $\mu \mathrm{m}$ thick) were obtained for each specimen and used for QD-IHC and conventional IHC staining. QD-IHC was similar to conventional IHC; the detailed procedures were described in our previous studies. ${ }^{14-18}$ Briefly, the sequence of the procedure was as follows: deparaffinizing, antigen retrieval, blocking $\left(2 \%\right.$ bovine serum albumin, $37^{\circ} \mathrm{C}$ for 30 minutes), incubation with primary antibody (dilution $1 / 100,37^{\circ} \mathrm{C}$ for 2 hours), washing, blocking, incubation with biotinylated secondary antibody (dilution 1/400, $37^{\circ} \mathrm{C}$ for 30 minutes), washing, blocking, application of QD-SA 605 probes (dilution $1 / 40,37^{\circ} \mathrm{C}$ for 30 minutes, emitting red light), washing, mounting, and observation (Olympus BX51 fluorescence microscope; Olympus
Corporation, Tokyo, Japan) with a blue light (wavelength of 450-490 nm) or a green light (wavelength of 510-515 $\mathrm{nm})$ excitation.

The interpretation of Ki67 staining and scoring were based on previous recommendations. ${ }^{11}$ For each specimen, a hot spot with many Ki67-positive cells was identified using a low-power field $(40 \times)$ on an Olympus BX51 microscope. Micrographs of medium-power fields $(100 \times, 200 \times)$, which included the aforementioned hot spot as well as a high-power field $(400 \times)$, were printed out. Three independent areas were selected in each spot. These three areas were saved as pictures and printed out. All cancer cells in the three micrographs were counted manually (1,000 cells were counted, Figure 2$)$. Two of the authors (JZS and CC) judged proportions of positive cells in a random order, independently, to generate the Ki67 score. The Ki67 score (\%) was defined as the number of Ki67-positive cells divided by the total number of cells counted, times 100 . 


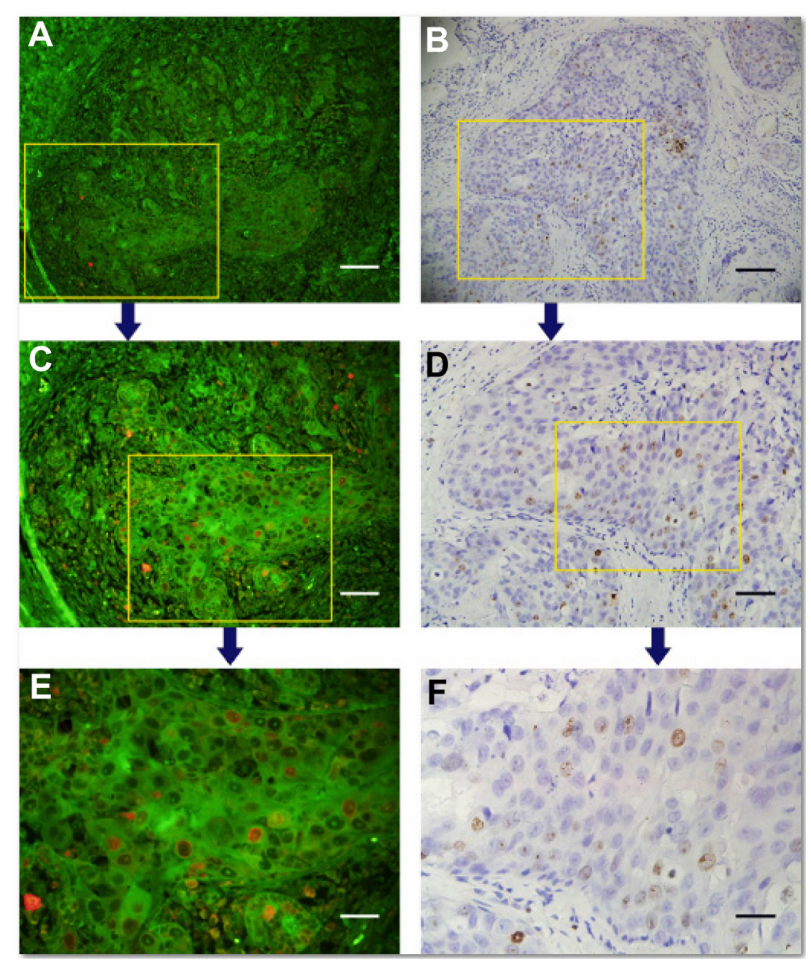

Figure 2 The staining and score of $\mathrm{Ki67}$ (MIBI) by QD-IHC and conventional IHC. Notes: A hot spot was identified using a low-power field (A and B, I0X) and sequentially enlarged ( $\mathbf{C}$ and $\mathbf{D}, 20 \times$; $\mathbf{E}$ and $\mathbf{F}, 40 \times$ ) to be selected for Ki67 scoring. Scale bar: $100 \mu \mathrm{m}$ (A and B), $50 \mu \mathrm{m}$ (C and D), $25 \mu \mathrm{m}$ (E and $\mathbf{F}$ ).

Abbreviations: IHC, immunohistochemistry; QD-IHC, quantum dotimmunohistochemistry.

\section{Statistical analysis}

The Pearson correlation and consistency $(\kappa)$ check were used to compare the results between QD-IHC and conventional IHC. The differences in Ki67 scores between the two pathologists were assessed using a two-sample $t$-test. Receiver-operating characteristic (ROC) curve analysis was conducted to evaluate the predictive value of the Ki67 score for 5-year disease-free survival. The optimal point with the highest sum value of sensitivity and specificity was defined as the cutoff. The Kaplan-Meier method was used to assess differences in 5-year disease-free survival (5-DFS). A multivariate Cox proportional hazards regression model was performed to analyze the independent prognostic factors. Statistical analyses were performed using SPSS 21.0 software (IBM Corporation, Armonk, NY, USA). Two-tailed $P<0.05$ was considered statistically significant.

\section{Results}

\section{Ki67 determination, assessment, and analysis}

In the feasibility study, Ki67 expression was observed at the same location in the nucleus by QD-IHC and IHC.
For accurate quantification, the mean of Ki67 scores generated by the two observers was used as the Ki67 score of the case. The Ki67 scores detected by QD-IHC and IHC were $7 \%-87 \%$ and $6 \%-83 \%$, respectively. Pearson correlation analysis showed that the correlation coefficient of the Ki67 scores for the two methods was 0.993 . The number of cases using different Ki67 score cutoffs showed good consistency between QD-IHC and IHC. For example, when we chose $30 \%$ as the cutoff, the same 70 cases were detected as positive by both methods ( $\kappa=0.874$, Kappa consistency check). The differences in Ki67 scores between the two observers were $2.08 \% \pm 1.54 \%$ using QD-IHC and $3.67 \% \pm 2.1 \%$ using IHC. The two-sample $t$-test showed a statistically significant difference $(t=-7.280, P<0.001)$.

\section{Ki67 expression in the HER2-positive (non-luminal) breast cancer cases}

Figure 3 shows the different Ki67 expressions as assessed by IHC and QD-IHC in the 108 HER2-positive (non-luminal) breast cancer cases. Means of the Ki67 scores by IHC and QD-IHC were 37.597\% (median: 34.5 ) and $40.227 \%$ (median: 36.5 ), respectively (Figure 4A). There were six cases with a Ki67 score $\leq 15 \%, 28$ cases $>15 \%$ and $\leq 30 \%, 37$ cases $>30 \%$ and $\leq 45 \%, 23$ cases $>45 \%$ and $\leq 60 \%$, and 14 cases $>60 \%$, as assessed by QD-IHC. IHC assessed nine, 28, 42, 16, and 13 cases in the respective categories (Figure 4B).

\section{ROC analysis of Ki67 scores by 5-DFS}

ROC analysis of the Ki67 scores by 5-DFS survival indicated that the Ki67 score could predict 5-DFS (Figure 4C).
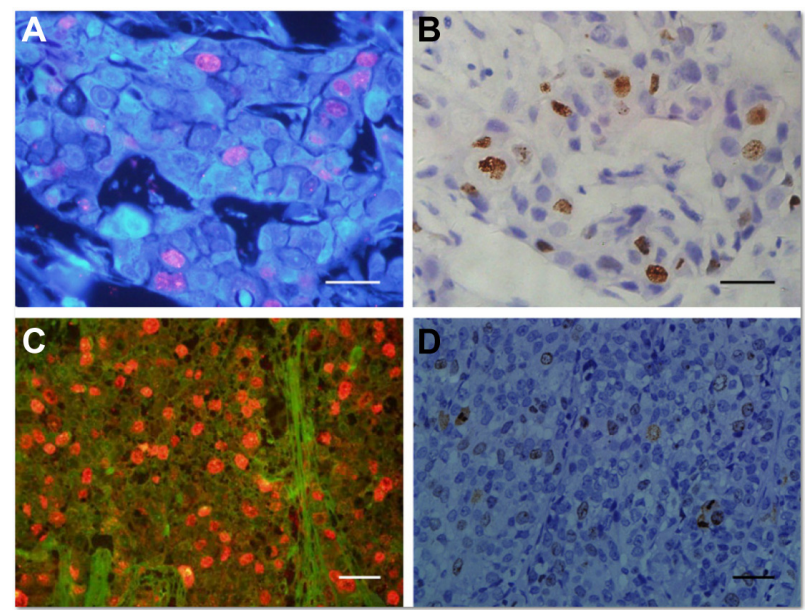

Figure 3 The different Ki67 expressions detected by QD-IHC (A,C) and IHC (B,D) in the same specimens.

Notes: (A and B) are the same specimens; (C and $\mathbf{D})$ are the same specimens. Scale bar: $25 \mu \mathrm{m}$.

Abbreviations: IHC, immunohistochemistry; QD-IHC, quantum dot-immunohistochemistry. 
A

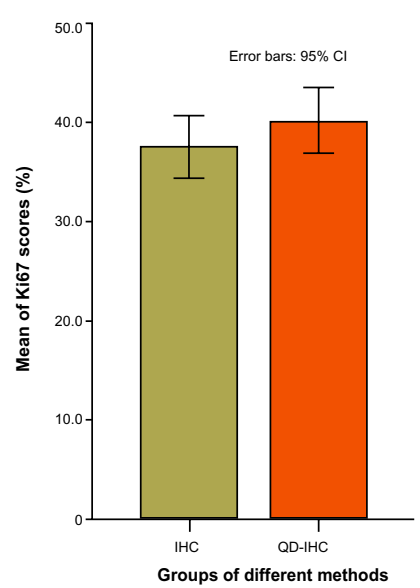

B

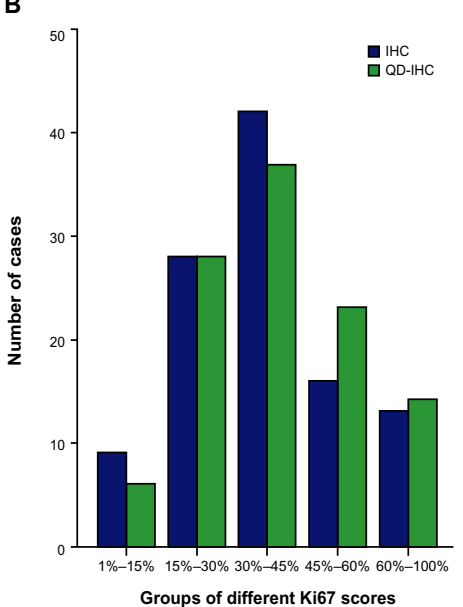

C

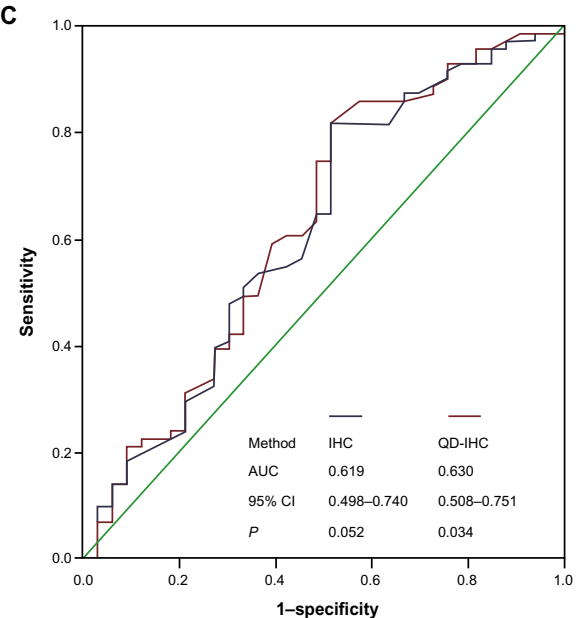

Figure 4 Mean of the Ki67 scores and their distribution in the HER2-positive (non-luminal) breast cancer cases by IHC and QD-IHC.

Notes: Mean of the Ki67 scores (A) and their distribution (B) in the HER2-positive (non-luminal) breast cancer cases by IHC and QD-IHC. Receiver-operating characteristic analysis of IHC and QD-IHC Ki67 scores by 5-DFS status of 108 cases (C).

Abbreviations: 5-DFS, 5-year disease free survival; IHC, immunohistochemistry; QD-IHC, quantum dot-immunohistochemistry; AUC, area under the curve; Cl, confidence interval.

According to the optimal sensitivity and specificity of the ROC curve by 5 -DFS status, we chose $30 \%$ as the cutoff for Ki67 scores, under which QD-IHC showed a sensitivity of $80.3 \%$ and a specificity of $51.4 \%$; IHC showed a sensitivity of $76.1 \%$ and a specificity of $51.4 \%$.

\section{Ki67 score and 5-DFS}

In this study, the 5-year disease-recurrence rate was 65.74\% (71/108), with 25 local recurrences and 46 distant recurrences. Based on the cutoff value of $30 \%$ for Ki67 scores, the 108 breast cancer tumors were classified into a high Ki67 expression subgroup and a low Ki67 expression subgroup. Using the QD-IHC Ki67 scores, the 5-year disease-recurrence rate was $76.32 \%$ in patients with high Ki67 expression $(n=76)$, and $40.63 \%$ in those with low Ki67 expression $(\mathrm{n}=32)$. The 5 -DFS of the two groups was significantly different ( $P=0.001, \log$-rank test, Figure $5 \mathrm{~A})$. Using the IHC Ki67 score, the five-year disease-recurrence rate was $74.65 \%$ in patients with high Ki67 expression $(n=71)$, and $48.65 \%$ in those with low Ki67 expression $(n=37)$. The 5 -DFS of the two groups was significantly different $(P=0.026$, log-rank test, Figure 5B).

The 5-year disease-recurrence rate and 5-DFS were further investigated according to lymph node status. In the lymph node-negative subgroup, the 5-DFS was statistically significantly different between patients with high and low Ki67 expressions, using both the QD-IHC Ki67 scores $(P=0.002)$ and the IHC Ki67 scores $(P=0.017)$ (Figure 5C and D). The QD-IHC Ki67 scores indicated a difference that was more statistically significant. There were no significant differences in 5-DFS between patients with high and low Ki67 expression using either method in lymph node-positive patients ( $P=0.532$ and $P=0.946, \log$-rank test, Figure 5E and F).

In multivariable analyses (Table 2), the Ki67 scores (cutoff 30\%) were predictive and the significance of the QDIHC Ki67 score was higher than of the IHC Ki67 score.

\section{Discussion}

In this study, QD-based immunofluorescent technology was used to detect and assess Ki67 expression. We found that QD-IHC showed good correlation and consistency with conventional IHC, with better image quality and sensitivity. This is similar to previous reports that detected other biomarkers using QD-IHC. ${ }^{14,16,18}$

Manual counting of as many as 1,000 cancer cells is frequently used to evaluate Ki67 in clinical practice, ${ }^{19-21}$ especially in developing countries. Automated counting by computer software is a candidate, but not all institutes can afford it and there is no evidence showing its superiority. ${ }^{6}$ Thus, manual counting was used to determine the Ki67 score by two observers respectively in our study. The QD-IHC Ki67 scores showed better agreement between the two observers than conventional IHC, possibly because the images were of better quality and brighter, which could have made manual counting easier. QD-IHC is more sensitive for detecting Ki67, more easily generates the Ki67 visual score, and is simpler in operation than conventional IHC; therefore, QD-IHC has the potential for clinical application in the future, especially in developing countries. Moreover, Ki67 QD-IHC may be 
A

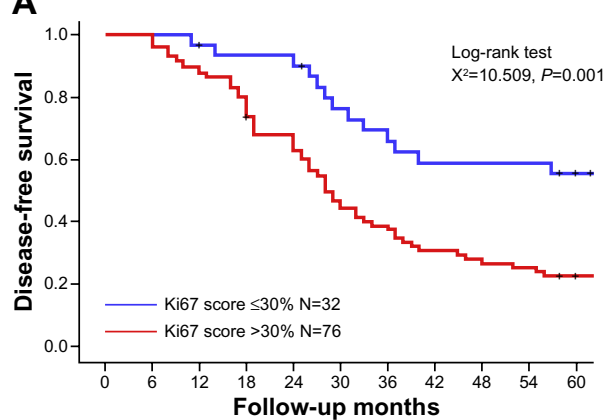

C

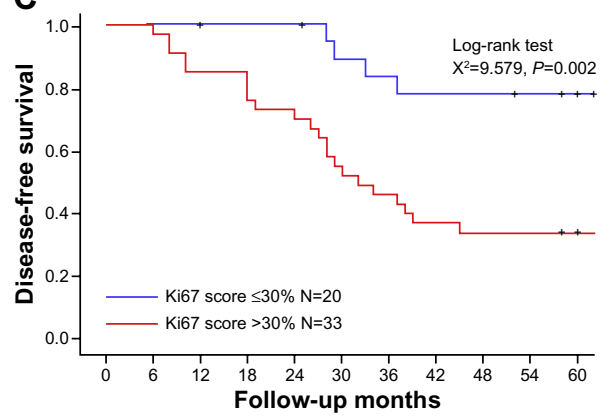

E

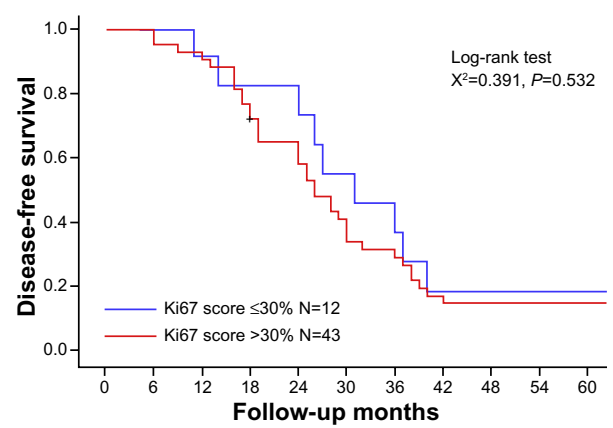

B

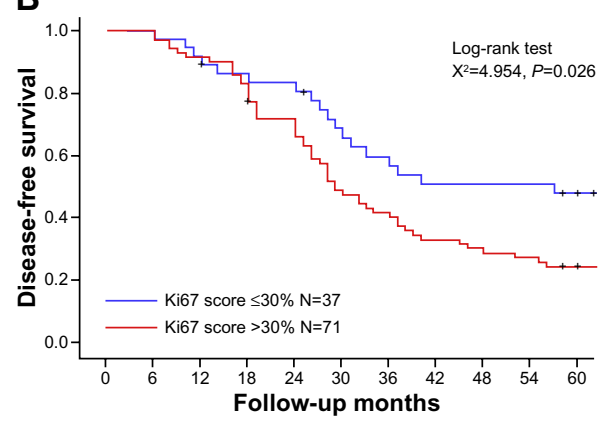

D

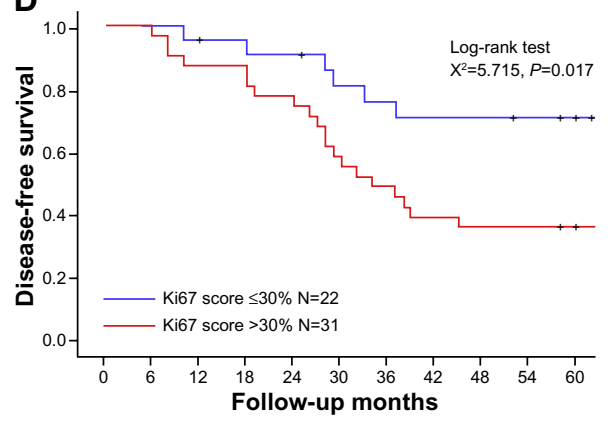

$\mathbf{F}$

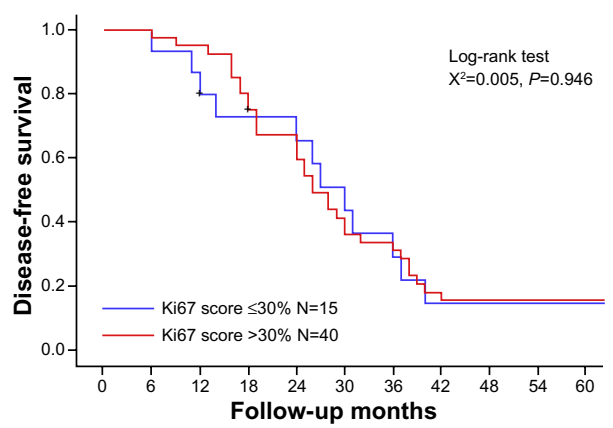

Figure 5 QD-IHC and IHC Ki67 scores and 5-DFS in HER2-positive (non-luminal) breast cancer cases and subgroups.

Note: The QD-IHC (A, C, and E) and IHC (B, D, and F) Ki67 scores and 5-DFS in all 108 HER2-positive (non-luminal) breast cancer cases (A and B), the lymph nodenegative subgroup ( $\mathbf{C}$ and $\mathbf{D})$, and the lymph node-positive subgroup ( $\mathbf{E}$ and $\mathbf{F})$.

Abbreviations: 5-DFS, 5-year disease free survival; IHC, immunohistochemistry; QD-IHC, quantum dot-immunohistochemistry; HER2, human epidermal growth factor receptor 2 .

more easily adapted to automated counting and quantitative analysis than IHC. Thus, as the function and features of Ki67 become better known and the standardization of QD-IHC across laboratories occurs, QD-IHC Ki67 score will progress further and become widely adopted in clinics.

For only HER2-positive (non-luminal) breast cancer in our study, the Ki67 expression is higher than previous reports. ${ }^{22}$ This result supported the view that HER2 overexpression is closely correlated with higher proliferation and aggressive behavior of breast cancer. However, the detailed relationship between Ki67 and HER2 expression remains unclear and requires further investigation.

Breast cancer has a highly variable prognosis for an individual patient. Key factors such as tumor size, histological grade, vascular invasion, and nodal status are helpful, but increasing attention is being paid to the molecular features of the tumor. ER, PR, and HER2 are now well established as predictive factors for treatment response and prognosis. Although not considered as an obligatory marker, Ki67 is also frequently measured, both as a static marker of proliferative activity and, by making multiple measurements during treatment, as a possible dynamic intermediate or surrogate marker of treatment efficacy. Even though many studies have demonstrated the prognostic value of Ki67, ${ }^{5}$ the debate on the prognostic role of Ki67 in breast cancer is still open. ${ }^{6}$ Almost all relevant studies were retrospective, and many of them included heterogeneous groups of patients who were treated and followed in various ways that were often incompletely documented. Furthermore, the assays for Ki67 were performed with different methods; cutoffs to designate 
Table 2 Multivariable analysis by 5-DFS

\begin{tabular}{|c|c|c|c|c|}
\hline \multirow[t]{2}{*}{ Items } & \multicolumn{2}{|l|}{ IHC Ki67 score* } & \multicolumn{2}{|c|}{ QD-IHC Ki67 score* } \\
\hline & HR (95\% Cl) & $P$-value & HR (95\% Cl) & $P$-value \\
\hline Age & $1.022(0.998-1.047)$ & 0.078 & $1.019(0.997-1.042)$ & 0.092 \\
\hline Tumor size & $2.579(1.796-3.704)$ & $<0.001$ & $2.629(1.853-3.786)$ & $<0.001$ \\
\hline Node status & $1.706(1.013-2.875)$ & 0.045 & $1.632(1.003-2.819)$ & 0.048 \\
\hline Tumor grade & $1.413(0.939-2.127)$ & 0.097 & $1.243(0.923-1.787)$ & 0.201 \\
\hline IHC Ki67 score* & 1.638 (1.084-2.995) & 0.029 & - & - \\
\hline QD-IHC Ki67 score* & - & - & 2.597 (1.299-5.192) & 0.007 \\
\hline
\end{tabular}

Note: *Cutoff, $30 \%$.

Abbreviations: 5-DFS, 5-year disease free survival; IHC, immunohistochemistry; QD-IHC, quantum dot-immunohistochemistry; HR, hazard ratio; Cl, confidence interval.

"positive" and "negative" or "high" and "low" Ki67 populations differ widely. In this study, to exclude the heterogeneity of breast cancer subtype and treatment, we chose only the HER2-positive (non-luminal) breast cancer subgroup. All patients had received modified radical mastectomy of breast cancer, and after the surgery, they only received doxorubicin-based chemotherapy; none of them received molecular targeted therapy. When we chose $30 \%$ as a cutoff, we found that, using either QD-IHC or IHC Ki67 scores, patients with high Ki67 expression had worse disease-free survival, especially in lymph node-negative patients. The multivariable analyses by 5-DFS also supported the view that the Ki67 score, determined by QD-IHC or IHC Ki67, was an independent predictor using this cutoff. These results suggested that $30 \%$ is a suitable cutoff in the HER2-positive (non-luminal) subgroup and the cutoff for the Ki67 score should be changed for breast cancer subgroups.

We also noted the recent research by Aleskandarany et $\mathrm{al} ;{ }^{23}$ they assessed the prognostic value of a proliferation assay using Ki67 IHC compared with mitotic count scores in the luminal, HER2-positive, and triple-negative biological classes of breast cancer. They found that neither mitotic count nor Ki67 score was associated with outcome in the HER2-positive or the triple-negative classes. However, the treatment given may not have been homogeneous in their study and the definition of subgroups in their study was different from ours. These factors could explain the differences in the results between our studies. Interestingly, Mrklić et al found that Ki67 has prognostic relevance in the triple-negative breast cancer subgroup. ${ }^{24}$ Considering the heterogeneity of the molecular subtypes that are defined by IHC, ${ }^{25}$ it is likely that the molecular taxonomy of breast cancers will evolve further.

This study has some limitations. First, it was a retrospective study using a nonrandomized database. Second, it included only 108 breast cancer specimens and the follow-up was only 5 years. Therefore, more accuracy could have been obtained with a larger sample size and longer follow-up. With the limited number of specimens, we did not divide lymph node-positive patients to N1, N2, and N3 (tumor node metastasis stage) subgroups; therefore, we only found a prognostic value of the Ki67 score in the lymph node-negative subgroups. Third, the Ki67 score has no uniform standard, ${ }^{26}$ and manual counting includes a subjective factor, which might have introduced some bias in the results. Nevertheless, QD-based nanotechnology provides a new insight into this elusive biomarker; this study demonstrated the prognostic value of Ki67 in the HER2-positive (non-luminal) breast cancer patients with equivalent treatment.

\section{Conclusion}

In conclusion, despite the above mentioned limitations, our study indicated that QD-based immunofluorescent imaging provided a new candidate method for Ki67 assessment; the Ki67 score was an independent prognosticator in HER2positive (non-luminal) breast cancer patients, especially those who were lymph node-negative.

\section{Acknowledgments}

This work was supported by grants from the National Science Foundation of China (Nos 81201196 and 81230031), the Fundamental Research Funds for the Central Universities (No 121004), the Key Scientific Research Project of Hubei's Provincial Department of Education (No D20126102), the Natural Science Foundation of Hubei Province, (Nos 301130851 and 2011CBD489), the Research Foundation of Public Health Bureau of Hubei Province (Nos JS-2011018, JX4B19 and JX3A14), and the National Science and Technology Major Project of the Ministry of Science and Technology of China (No 2012YQ16020306).

\section{Disclosure}

The authors declare no conflicts of interest in this work. 


\section{References}

1. Jemal A, Bray F, Center MM, Ferlay J, Ward E, Forman D. Global cancer statistics. CA Cancer J Clin. 2011;61(2):69-90.

2. Gerdes J, Lemke H, Baisch H, Wacker HH, Schwab U, Stein H. Cell cycle analysis of a cell proliferation-associated human nuclear antigen defined by the monoclonal antibody Ki-67. J Immunol. 1984;133(4): 1710-1715.

3. Gerdes J, Li L, Schlueter C, et al. Immunobiochemical and molecular biologic characterization of the cell proliferation-associated nuclear antigen that is defined by monoclonal antibody Ki-67. Am J Pathol. 1991;138(4):867-873.

4. Gerdes J, Schwab U, Lemke H, Stein H. Production of a mouse monoclonal antibody reactive with a human nuclear antigen associated with cell proliferation. Int J Cancer. 1983;31(1):13-20.

5. Yerushalmi R, Woods R, Ravdin PM, Hayes MM, Gelmon KA. Ki67 in breast cancer: prognostic and predictive potential. Lancet Oncol. 2010;11(2):174-183.

6. Kontzoglou K, Palla V, Karaolanis G, et al. Correlation between Ki67 and breast cancer prognosis. Oncology. 2013;84(4):219-225.

7. Cheang MC, Chia SK, Voduc D, et al. Ki67 index, HER2 status, and prognosis of patients with luminal B breast cancer. J Natl Cancer Inst. 2009;101(10):736-750.

8. Goldhirsch A, Wood WC, Coates AS, Gelber RD, Thürlimann B, Senn HJ; Panel members. Strategies for subtypes - dealing with the diversity of breast cancer: highlights of the St Gallen International Expert Consensus on the Primary Therapy of Early Breast Cancer 2011. Ann Oncol. 2011;22(8):1736-1747.

9. Cuzick J, Dowsett M, Pineda S, et al. Prognostic value of a combined estrogen receptor, progesterone receptor, $\mathrm{Ki}-67$, and human epidermal growth factor receptor 2 immunohistochemical score and comparison with the Genomic Health recurrence score in early breast cancer. J Clin Oncol. 2011;29(32):4273-4278.

10. Pathmanathan N, Balleine RL. Ki67 and proliferation in breast cancer. J Clin Pathol. 2013;66(6):512-516.

11. Dowsett M, Nielsen TO, A'Hern R, et al; International Ki-67 in Breast Cancer Working Group. Assessment of Ki67 in breast cancer: recommendations from the International Ki67 in Breast Cancer working group. J Natl Cancer Inst. 2011;103(22):1656-1664.

12. Azzazy HM, Mansour MM, Kazmierczak SC. From diagnostics to therapy: prospects of quantum dots. Clin Biochem. 2007;40(13-14): 917-927.

13. Chen C, Peng J, Sun SR, Peng CW, Li Y, Pang DW. Tapping the potential of quantum dots for personalized oncology: current status and future perspectives. Nanomedicine (Lond). 2012;7(3): 411-428.
14. Chen C, Peng J, Xia HS, et al. Quantum dots-based immunofluorescence technology for the quantitative determination of HER2 expression in breast cancer. Biomaterials. 2009;30(15):2912-2918.

15. Chen C, Xia HS, Gong YP, et al. The quantitative detection of total HER2 load by quantum dots and the identification of a new subtype of breast cancer with different 5-year prognosis. Biomaterials. 2010;31(33):8818-8825.

16. Chen C, Peng J, Xia H, et al. Quantum-dot-based immunofluorescent imaging of HER2 and ER provides new insights into breast cancer heterogeneity. Nanotechnology. 2010;21(9):95101.

17. Chen C, Sun SR, Gong YP, et al. Quantum dots-based molecular classification of breast cancer by quantitative spectroanalysis of hormone receptors and HER2. Biomaterials. 2011;32(30):7592-7599.

18. Yang XQ, Chen C, Peng CW, et al. Quantum dot-based quantitative immunofluorescence detection and spectrum analysis of epidermal growth factor receptor in breast cancer tissue arrays. Int J Nanomedicine. 2011;6:2265-2273.

19. Trihia H, Murray S, Price K, et al; International Breast Cancer Study Group. Ki-67 expression in breast carcinoma: its association with grading systems, clinical parameters, and other prognostic factors a surrogate marker? Cancer. 2003;97(5):1321-1331.

20. Viale G, Giobbie-Hurder A, Regan MM, et al; Breast International Group Trial 1-98.. Prognostic and predictive value of centrally reviewed Ki-67 labeling index in postmenopausal women with endocrineresponsive breast cancer: results from Breast International Group Trial 1-98 comparing adjuvant tamoxifen with letrozole. J Clin Oncol. 2008;26(34):5569-5575.

21. Endo Y, Toyama T, Takahashi S, et al. High estrogen receptor expression and low Ki67 expression are associated with improved time to progression during first-line endocrine therapy with aromatase inhibitors in breast cancer. Int J Clin Oncol. 2011;16(5):512-518.

22. Haroon S, Hashmi AA, Khurshid A, et al. Ki67 index in breast cancer: correlation with other prognostic markers and potential in pakistani patients. Asian Pac J Cancer Prev. 2013;14(7):4353-4358.

23. Aleskandarany MA, Green AR, Benhasouna AA, et al. Prognostic value of proliferation assay in the luminal, HER2-positive, and triple-negative biologic classes of breast cancer. Breast Cancer Res. 2012;14(1):R3.

24. Mrklić I, Ćapkun V, Pogorelić Z, Tomić S. Prognostic value of Ki-67 proliferating index in triple negative breast carcinomas. Pathol Res Pract. 2013;209(5):296-301.

25. Guiu S, Michiels S, André F, et al. Molecular subclasses of breast cancer: how do we define them? The IMPAKT 2012 Working Group Statement. Ann Oncol. 2012;23(12):2997-3006.

26. Pathmanathan N, Balleine RL. Ki67 and proliferation in breast cancer. J Clin Pathol. 2013;66(6):512-516.
International Journal of Nanomedicine

\section{Publish your work in this journal}

The International Journal of Nanomedicine is an international, peerreviewed journal focusing on the application of nanotechnology in diagnostics, therapeutics, and drug delivery systems throughout the biomedical field. This journal is indexed on PubMed Central, MedLine, CAS, SciSearch $\AA$, Current Contents ${ }^{\circledR} /$ Clinical Medicine,

\section{Dovepress}

Journal Citation Reports/Science Edition, EMBase, Scopus and the Elsevier Bibliographic databases. The manuscript management system is completely online and includes a very quick and fair peer-review system, which is all easy to use. Visit http://www.dovepress.com/ testimonials.php to read real quotes from published authors. 